\title{
Synthesis and Homologation of an Azetidin-2-yl Boronic Ester with $\alpha$ - Lithioalkyl Triisopropylbenzoates
}

\author{
Pascal K. Delany and David M. Hodgson*
}

Department of Chemistry, Chemistry Research Laboratory, University of Oxford, Mansfield Road, Oxford OX1 3TA, U.K.

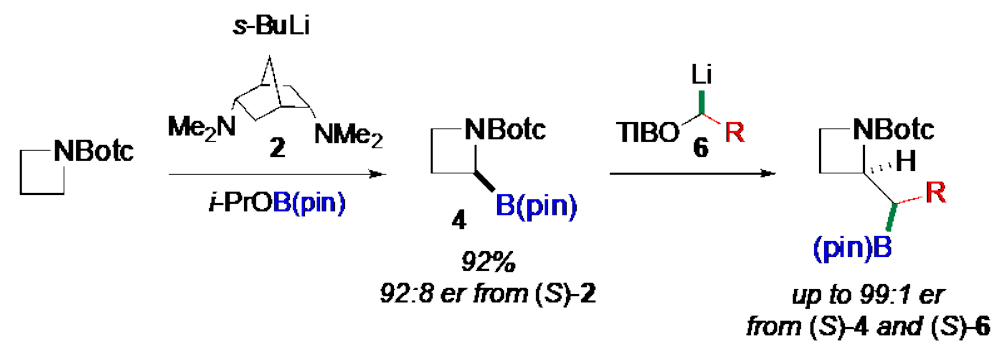

ABSTRACT: An $\alpha$-boryl azetidine, obtained by $\alpha$-lithiation-borylation of $N$-Botc azetidine, undergoes reaction with
$\alpha$-triisopropylbenzoyloxy organolithiums to give homologated boronic esters that can be further oxidised, homologated, arylated
and deprotected to give a range of $\alpha$-substituted azetidines. Scalemic $\alpha$-boryl azetidine- $\alpha$-triisopropylbenzoyloxy organolithium
pairings show stereospecific reagent control, providing access to either diastereomeric series of homologated boronic esters with
very high
ers.

Saturated azacycles are ubiquitous structural motifs in natural products, ${ }^{1}$ drug designs $^{2}$ and organocatalysts. ${ }^{3}$ Synthetic studies on pyrrolidines ${ }^{4}$ and piperidines ${ }^{5}$ have been extensive and aziridines ${ }^{6}$ have also seen more attention compared with azetidines. However, with azetidine moieties increasingly emerging as constituents of highly bioactive compounds, ${ }^{7}$ developing effective syntheses of substituted azetidines has become an important and growing field of chemistry. $^{8}$

Our previous research on azetidine $\alpha$-lithiation-electrophile trapping gave 2-substituted azetidines, through the use of a thiopivaloyl or tert-butoxythiocarbonyl (Botc) Nprotecting/activating group (Scheme 1, A). ${ }^{9-11}$ The methodology enables direct diversifying $\alpha$-functionalisation of the azetidine moiety. Good enantioselectivities can be achieved by $\alpha$-lithiation of $N$-Botc azetidine 1 in the presence of the chiral DIANANE ligand $2^{12}$ and trapping with certain electrophiles (eg, Scheme 1, A). ${ }^{10,13}$ However, reactions with aldehydes typically suffered from poor diastereoselectivity and enolisable aldehydes, such as hydrocinnamaldehyde, gave no conversion.

Boronic esters are established as highly versatile functional groups, known to undergo many stereospecific transformations. ${ }^{14}$ In a recent synthesis of (-)-stemaphylline, Aggarwal, Leonori and co-workers reported the preparation and some $\mathrm{C}-\mathrm{C}$ bond-forming reactions of 2-B(pin)-pyrrolidine 3 (eg, Scheme 1, B). ${ }^{15}$ We considered that if access to 2-B(pin)-azetidine 4 was achievable (Scheme 1, C), then this could lead on to providing a broader range of $\alpha$-functionalised azetidines than previously possible through direct $\alpha$-lithiation- electrophile trapping. Here we communicate progress on these areas, in both racemic and asymmetric variants.

Scheme 1. $\alpha$-Substitution of azetidines and pyrrolidines through organolithium chemistry

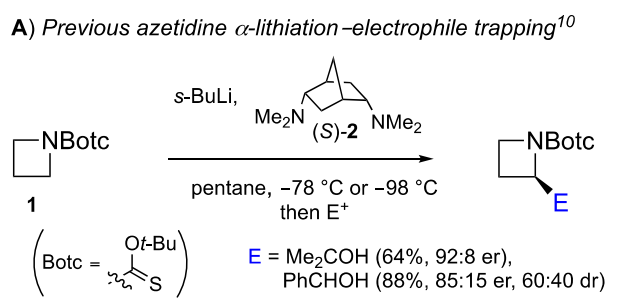

B) Aggarwal's homologation of N-Boc 2-B(pin)-pyrrolidine (3) ${ }^{15}$
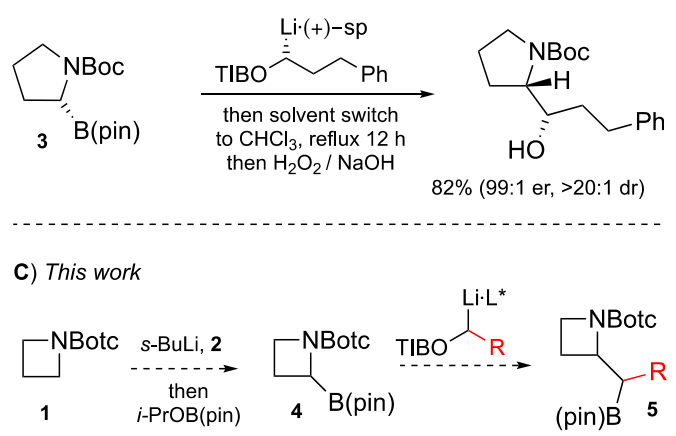

We began by applying our azetidine $\alpha$-lithiation chemistry in a racemic sense with $N$-Botc azetidine 1 and TMEDA in $\mathrm{THF},{ }^{10}$ using $i$-PrOB(pin) as the electrophile; this gave boronic 
ester 3 in moderate yield (53\%, Scheme 2). A factor contributing to the modest yield was likely product loss during purification by column chromatography. Despite testing a variety of different deactivated silica gels, chromatographed boronic ester 3 proved impossible to isolate without partial decomposition. ${ }^{16}{ }^{1} \mathrm{H}$ NMR analysis of the crude material indicated pinacol as a major impurity, which could not be easily removed via aqueous wash. It was reasoned that running the reaction in a more hydrophobic solvent than THF, such as pentane, could enable easier removal of impurities such as pinacol during aqueous work-up. Conditions for lithiation/electrophile trapping in pentane using $(S)-\mathbf{2}$ as a ligand (Scheme 1, A) ${ }^{10}$ were applied with $( \pm)$-2, to give 2B(pin)-azetidine 4 in excellent yield (89\%) and sufficiently high purity to negate the need for chromatographic purification. Following acid/base extraction the diamine ligand could also be efficiently recovered $(88 \%)$ and recycled.

Scheme 2. Racemic and asymmetric $\alpha$-lithiationborylation of $N$-Botc azetidine 1

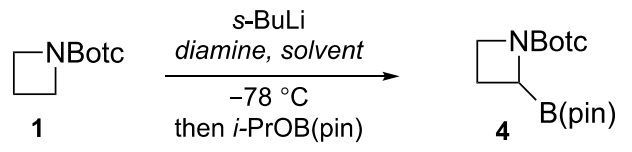

$53 \%$ (using TMEDA in THF) $89 \%$ (using ( \pm )-2 in pentane) $92 \%, 92: 8$ er (using $(S)-2$ in pentane)

Applying the above conditions using (S)-2 gave the enantioenriched boronic ester (+)-4 also in excellent yield $(92 \%)$ and high enantioenrichment (92:8 er). The absolute configuration of boronic ester ( + - 4 was determined to be $S$ by derivatisation to an alcohol enantiomeric to that derived from $(S)$-azetidine-2-carboxylic acid. ${ }^{16}$ The sense of asymmetric induction is opposite to that previously seen with MeI, ${ }^{10}$ but the same as found for acetone and benzaldehyde. ${ }^{13}$ These findings parallel observations by O'Brien and co-workers, who noted that the sense of asymmetric induction following lithiation of $N$-thiopivaloyl azetidine in the presence of (-)sparteine is dependent on the nature of the electrophile. ${ }^{17}$

An attractive functionalisation of boronic ester 4 would be homologation via ate complex formation with an $\alpha$-triisopropylbenzoyloxy (TIBO) organolithium and subsequent 1,2-metallate rearrangement ( $c f$, Scheme 1, B and C). ${ }^{18}$ This should in principle allow assembly of two contiguous stereocenters with, ultimately, control arising from choice of reactant configurations. Homologations of $\alpha$-tonitrogen boronic esters have often been found to be difficult, ${ }^{15,19}$ although such transformations can benefit from a solvent exchange to chloroform following ate complex formation, to facilitate the migration step. ${ }^{15,20}$ Following such protocol, 2-B(pin)-azetidine $( \pm)-4$ was reacted with $\alpha$ lithiobenzoate $( \pm)$-6a ( 1.3 equiv) to give homologated boronic ester 5a in moderate yield (45\%, Scheme 3$)$, as a readily separable mixture of diastereomers $(74: 26 \mathrm{dr}$, major diastereomer shown $\left.{ }^{16}\right)$. Using boronic ester 4 in slight excess (1.5 equiv) significantly improved the yield of $\mathbf{5 a}(71 \%, 83: 17$ dr, Scheme 3). While these latter conditions would be appropriate when the benzoate is the more precious material, studies to establish an efficient method for homologation with boronic ester $\mathbf{4}$ as the limiting reagent were also undertaken. Aggarwal and co-workers previously employed stannanes in a diamine-free tin-lithium exchange approach to $\alpha$-lithiated benzoates and carbamates. ${ }^{21}$ This latter method has the benefit of reduced steric hindrance around $\alpha$-TIBO organolithiums such as $\mathbf{6 b}$, as the only coordinating species on their generation is solvent $\left(\mathrm{Et}_{2} \mathrm{O}\right)$. Pleasingly, following this strategy with 1.3 equiv of tributylstannyl-derived organolithium $( \pm)-\mathbf{6 b}$ gave the homologated boronic ester 5a in good yield (69\%, 72:28 dr, Scheme 3).

Scheme 3. Homologation of 2-B(pin)-azetidine ( \pm )-4

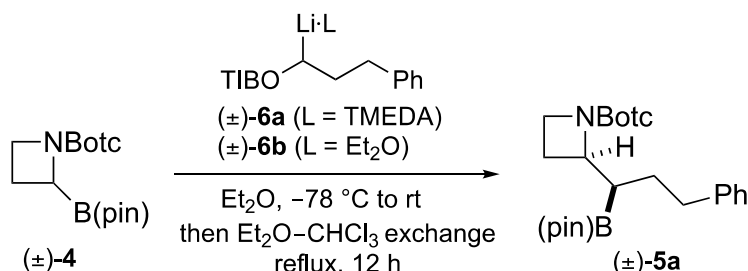

$45 \%, 74: 26$ dr (from 4:6a, 1:1.3)

$71 \%, 83: 17$ dr (from 4:6a, 1.5:1)

$69 \%, 72: 28$ dr (from 4:6b, 1:1.3)

Having established conditions for homologation of azetidine boronic ester 4 where it could be used as the limiting reagent, a study of substrate scope with respect to the stannyl-derived $\alpha$-TIBO organolithium was carried out, to examine reaction tolerance towards steric hindrance and functional groups. Reaction using a smaller $\alpha$-TIBO ethyl stannane proceeded smoothly; a shorter reflux time of $3 \mathrm{~h}$ was sufficient, to give homologated boronic ester $\mathbf{5 b}$ in $68 \%$ yield (Table 1 , entry 1 ). Applying this shorter reflux time with $\alpha$-TIBO organolithium 6b also gave 5a in $65 \%$ yield and unchanged $\mathrm{dr}$. More sterically demanding $\beta$-branched stannanes remained effective, giving homologated isobutyl and methylcyclopentyl boronic esters $\mathbf{5 c}$ and $\mathbf{5 d}$ in good yields (Table 1, entries 2 and $3)$. However, further increase of the steric bulk at the $\beta$ position, using an $\alpha$-TIBO neopentyl stannane, failed to give any of the desired homologated product 5e (entry 4). In this last case, isolation of the $\mathrm{B}(\mathrm{pin})$ neopentyl benzoate $(71 \%)^{16}$ from overall tributyltin-B(pin) exchange suggests generation of the intermediate ate complex proceeded, but its collapse occurred without 1,2-metallate rearrangement. Homologations performed using stannanes bearing alkenyl, silyloxy and ketal functionality all proved viable (entries 5-7).

Table 1. Homologation scope of 2-B(pin)-azetidine 4 with stannane-derived $\alpha$-TIBO organolithiums
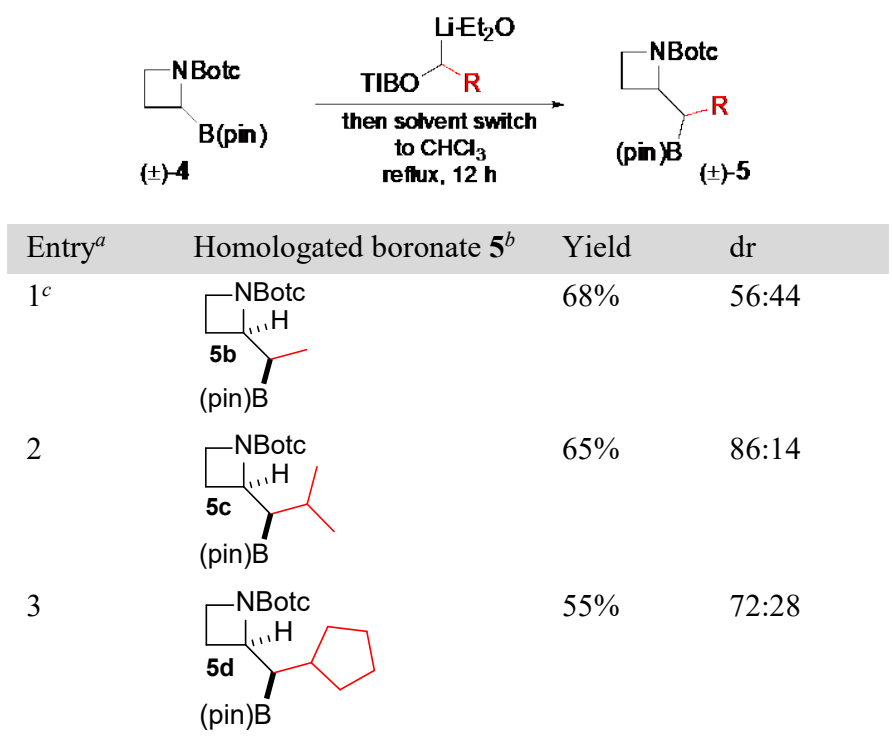


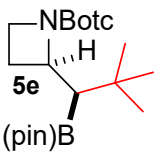

5

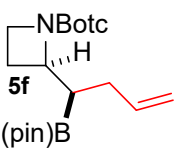

$6^{e}$

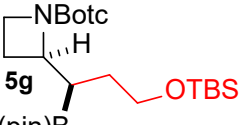

$7 f$

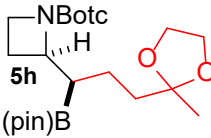

$56 \%$

$67: 33$

${ }^{a}$ Unless otherwise noted, (i) reactions used 2-B(pin)-azetidine 4 $(0.42 \mathrm{mmol})$, stannane $(0.55 \mathrm{mmol}), 12 \mathrm{~h}$ reflux in $\mathrm{CHCl}_{3}$ and (ii) amount of individual diastereomers isolated after chromatography used to give drs and combined yields. ${ }^{b}$ Major diastereomer shown (5c,d,f-h assigned by analogy to $\mathbf{5 a}, \mathbf{b}) .{ }^{16}{ }^{c} 3 \mathrm{~h}$ reflux. ${ }^{d}$ Tin-B(pin) exchange observed (71\%). ${ }^{16}{ }^{e}$ Using 2-B(pin)-azetidine $4(0.21$ $\mathrm{mmol})$ and stannane $(0.28 \mathrm{mmol})$. ${ }^{f}$ Yield for inseparable diastereomeric mixture and $\mathrm{dr}$ from ${ }^{1} \mathrm{H}$ NMR analysis.

The observation of diastereoselectivity in the above boronate homologations suggests possible matched/mismatched effects depending on reactants configurations. ${ }^{22}$ This could potentially render the asymmetric approach less effective in enabling access to the enantiomers of both diastereomers. To examine this aspect, separate reactions were carried out (Scheme 4 ) of $S$ and $R$ boronic ester $\mathbf{4}$ with $\alpha$-lithiobenzoate $(S)-\mathbf{6 b}$ [generated by stereoretentive $\mathrm{Sn}-\mathrm{Li}$ exchange ${ }^{23}$ from the corresponding $S$ stannane $(95: 5$ er ${ }^{18}$. Pleasingly, both reactions proceeded to give homologated boronic esters $(R, R)$ - and $(S, R)$-5a respectively in high diastereoselectivities and excellent enantioselectivities (up to 99:1 er), albeit with slightly lower yields compared with the racemic homologation. Similar selectivities (up to 99:1 er) and yields were also achieved for $\mathbf{5 b} .^{16}$

\section{Scheme 4. Asymmetric homologation of $S$ and $R$ boronic} ester 4

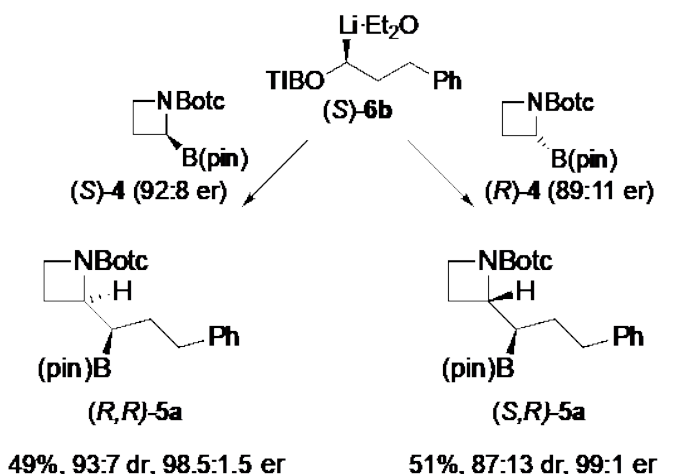

To demonstrate synthetic versatility of the homologated boronic esters, further transformations of boronic ester $\mathbf{5 a}$ were performed [Scheme 5, shown for $\left(R^{*}, R^{*}\right)-\mathbf{5 a}$; separately examined $\left(R^{*}, S^{*}\right)-\mathbf{5 a}$ behaved similarly $\left.{ }^{16}\right]$. Oxidation of boronic ester $\mathbf{5 a}$ to alcohol 7 was achieved using sodium perborate in good yield (66\%). Matteson homologation, using dibromomethane, successfully gave the one-carbon homologated boronic ester 8 in high yield (79\%). Arylation was achieved using DDQ as the activating electrophile, ${ }^{24}$ to give the furanylated product 9 in moderate yield (55\%). This reaction also resulted in the formation of homoallylic amine $10(28 \%)$. The latter likely arises from a competitive 1,2elimination/ring-opening pathway, indicating that these $\mathrm{C}-\mathrm{B}$ derivatisations, on a system with a $\beta$-electron withdrawing functional group incorporated within a strained ring, are not always straightforward. Finally, Botc deprotection of 5a in ethereal $\mathrm{HCl}$ gave the azetidinium chloride salt $\mathbf{1 1}$ (quant), which further demonstrates the utility/lability of this recently introduced protecting/activating group. ${ }^{10}$ Whiting and coworkers have shown homoboroprolines to be effective organocatalysts for asymmetric aldol reactions, ${ }^{19}$ and the current formation of a similar azetidinium boronic ester could lead to applications in this area.

\section{Scheme 5. Transformations of homologated boronic ester} $\left(R^{*}, R^{*}\right)-5 a$

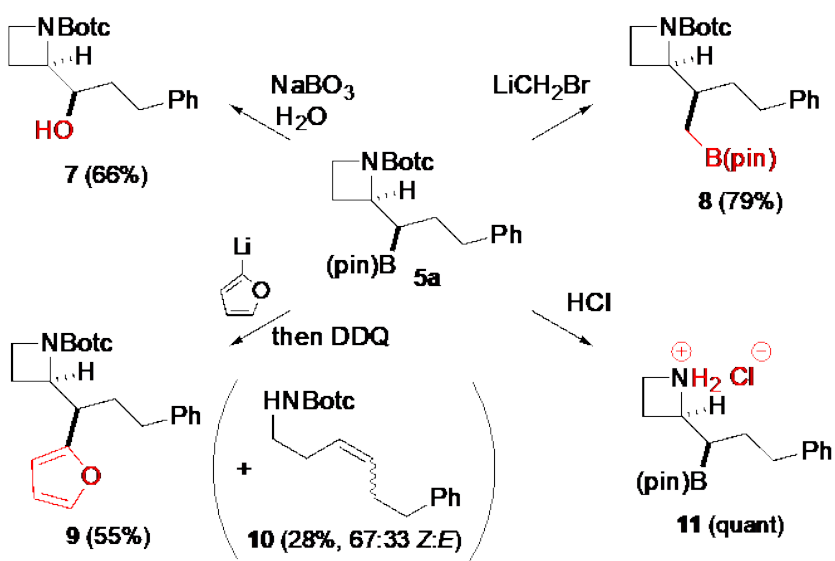

In conclusion, we have developed a synthetic route to enantioenriched 2-B(pin)-azetidine $\mathbf{4}$ and the latter has been converted to homologated azetidine boronic esters, by boronate formation with $\alpha$-lithiobenzoates and subsequent 1,2metallate rearrangement. This process can be performed with high diastereo and enantioselectivity. The homologated boronic esters can be further transformed into $\alpha$-substituted azetidines which cannot be accessed through direct $\alpha$ lithiation-electrophile trapping chemistry.

\section{ASSOCIATED CONTENT}

\section{Supporting Information}

Full experimental procedures and characterization data. This material is available free of charge via the Internet at http://pubs.acs.org.

\section{AUTHOR INFORMATION}

\section{Corresponding Author}

*E-mail: david.hodgson@chem.ox.ac.uk

\section{Notes}

The authors declare no competing financial interest.

\section{ACKNOWLEDGMENT}

We thank the EPSRC for studentship support (to P.K.D.).

\section{REFERENCES}

(1) O'Hagan, D. Pyrrole, Pyrrolidine, Pyridine, Piperidine and Tropane Alkaloids. Nat. Prod. Rep. 2000, 17, 435-446. 
(2) (a) Taylor, R. D.; MacCoss, M.; Lawson, A. D. G. Rings in Drugs. J. Med. Chem. 2014, 57, 5845-5859. (b) Vitaku, E.; Smith, D. T.; Njardarson, J. T. Analysis of the Structural Diversity, Substitution Patterns, and Frequency of Nitrogen Heterocycles among U.S. FDA Approved Pharmaceuticals. J. Med. Chem. 2014, 57, 10257-10274.

(3) (a) Dondoni, A.; Massi, A. Asymmetric Organocatalysis: from Infancy to Adolescence. Angew. Chem., Int. Ed. 2008, 47, 46384660. (b) Liu, J.; Wang, L. Recent Advances in Asymmetric Reactions Catalyzed by Proline and Its Derivatives. Synthesis 2017, 49, 960-972.

(4) Bhat, C.; Tilve, S. G. Recent Advances in the Synthesis of Naturally Occurring Pyrrolidines, Pyrrolizidines and Indolizidine Alkaloids using Proline as a Unique Chiral Synthon. RSC Adv. 2014, 4, 5405-5452.

(5) (a) Buffat, M. G. P. Synthesis of Piperidines. Tetrahedron 2004, 60, 1701-1729. (b) Källström, S.; Leino, R. Synthesis of Pharmaceutically Active Compounds containing a Disubstituted Piperidine Framework. Bioorg. Med. Chem. 2008, 16, 601-635.

(6) (a) Pellissier, H. Recent Developments in Asymmetric Aziridination. Adv. Synth. Catal. 2014, 356, 1899-1935. (b) Macha, L.; D'hooghe, M.; Ha, H. J. Deployment of Aziridines for the Synthesis of Alkaloids and Their Derivatives. Synthesis 2019, 51, $1491-1515$

(7) (a) Keith, J. M.; Jones, W. M.; Pierce, J. M.; Seierstad, M.; Palmer, J. A.; Webb, M.; Karbarz, M. J.; Scott, B. P.; Wilson, S. J.; Luo, L.; Wennerholm, M. L.; Chang, L.; Brown, S. M.; Rizzolio, M.; Rynberg, R.; Chaplan, S. R.; Breitenbucher, J. G. Heteroarylureas with Spirocyclic Diamine Cores as Inhibitors of Fatty Acid Amide Hydrolase. Bioorg. Med. Chem. Lett. 2014, 24, 737-741. (b) Han, M.; Song, C.; Jeong, N.; Hahn, H. G. Exploration of 3-Aminoazetidines as Triple Reuptake Inhibitors by Bioisosteric Modification of 3- $\alpha$ Oxyazetidine. ACS Med. Chem. Lett. 2014, 5, 999-1004. (c) Maetani, M.; Kato, N.; Jabor, V. A. P.; Calil, F. A.; Nonato, M. C.; Scherer, C. A.; Schreiber, S. L. Discovery of Antimalarial Azetidine-2carbonitriles that Inhibit P. falciparum Dihydroorotate Dehydrogenase. ACS Med. Chem. Lett. 2017, 8, 438-442. (d) Maetani, M.; Zoller, J.; Melillo, B.; Verho, O.; Kato, N.; Pu, J.; Comer, E.; Schreiber, S. L. Synthesis of a Bicyclic Azetidine with In Vivo Antimalarial Activity Enabled by Stereospecific, Directed C( $\left.\mathrm{sp}^{3}\right)$-H Arylation. J. Am. Chem. Soc. 2017, 139, 11300-11306.

(8) (a) Mehra, V.; Lumb, I.; Anand, A.; Kumar, V. Recent Advances in Synthetic Facets of Immensely Reactive Azetidines. RSC $A d v$. 2017, 7, 45763-45783. (b) Antermite, D.; Degennaro, L.; Luisi, R. Recent Advances in the Chemistry of Metallated Azetidines. Org. Biomol. Chem. 2017, 15, 34-50. (c) Reidl, T. W.; Anderson, L. L. Divergent Functionalizations of Azetidines and Unsaturated Azetidines. Asian. J. Org. Chem. 2019, 8, 931-945.

(9) Hodgson, D. M.; Kloesges, J. Lithiation-Electrophilic Substitution of $N$-Thiopivaloylazetidine. Angew. Chem., Int. Ed. 2010, 49, 2900-2903.

(10) Hodgson, D. M.; Mortimer, C. L.; McKenna, J. M. Amine Protection $/ \alpha$-Activation with the tert-Butoxythiocarbonyl Group: Application to Azetidine Lithiation-Electrophilic Substitution. Org. Lett. 2015, 17, 330-333.

(11) Jackson, K. E.; Mortimer, C. L.; Odell, B.; McKenna, J. M.; Claridge, T. D. W.; Paton, R. S.; Hodgson, D. M. $\alpha$ - and $\alpha^{\prime}-$ Lithiation-Electrophile Trapping of $N$-Thiopivaloyl and $N$-tertButoxythiocarbonyl $\alpha$-Substituted Azetidines: Rationalization of the Regiodivergence Using NMR and Computation. J. Org. Chem. 2015, 80, 9838-9846.

(12) Praz, J.; Guénée, L.; Aziz, S.; Berkessel, A.; Alexakis, A. Evaluation of the Chiral DIANANE Backbone as Ligand for Organolithium Reagents. Adv. Synth. Catal. 2012, 354, 1780-1790.

(13) The stereochemistry of acetone- and benzaldehyde-trapped azetidines has been reassigned from that indicated in our earlier study (Ref. 10). See Supporting Information for details.

(14) (a) Hall, D. G. Boronic Acids: Preparation and Applications in Organic Synthesis, Medicine and Materials; Wiley-VCH: Weinheim, 2011. (b) Andrés, P.; Ballano, G.; Calaza, M. I.; Cativiela, C. Synthesis of $\alpha$-Aminoboronic Acids. Chem. Soc. Rev. 2016, 45, 2291-2307. (c) Sandford, C.; Aggarwal, V. K. Stereospecific Functionalizations and Transformations of Secondary and Tertiary Boronic Esters. Chem. Commun. 2017, 53, 5481-5494.
(15) Varela, A.; Garve, L. K. B.; Leonori, D.; Aggarwal, V. K. Stereocontrolled Total Synthesis of (-)-Stemaphylline. Angew. Chem., Int. Ed. 2017, 56, 2127-2131.

(16) See Supporting Information for details.

(17) Rayner, P. J.; Smith, J. C.; Denneval, C.; O’Brien, P.; Clarke, P. A.; Horan, R. A. J. Mechanistic Interrogation of the Asymmetric Lithiation-Trapping of $N$-Thiopivaloyl Azetidine and Pyrrolidine. Chem. Commun. 2016, 52, 1354-1357.

(18) Larouche-Gauthier, R.; Fletcher, C. J.; Couto, I.; Aggarwal, V. $\mathrm{K}$. Use of Alkyl 2,4,6-Triisopropylbenzoates in the Asymmetric Homologation of Challenging Boronic Esters. Chem. Commun. 2011, 47, 12592-12594.

(19) K. Arnold, K.; Batsanov, A. S.; Davies, B.; Grosjean, C.; Schuetz, T.; Whiting, A.; Zawatzky, K. The First Example of Enamine-Lewis Acid Cooperative Bifunctional Catalysis: Application to the Asymmetric Aldol Reaction. Chem. Commun. 2008, 33, 3879-3881.

(20) Roesner, S.; Casatejada, J. M.; Elford, T. G.; Sonawane, R. P.; Aggarwal, V. K. Enantioselective Syntheses of (+)-Sertraline and (+)Indatraline Using Lithiation/Borylation-Protodeboronation Methodology. Org. Lett. 2011, 13, 5740-5743.

(21) (a) Blair, D. J.; Fletcher, C. J.; Wheelhouse, M. P.; Aggarwal, V. K. Stereocontrolled Synthesis of Adjacent Acyclic QuaternaryTertiary Motifs: Application to a Concise Total Synthesis of (-)Filiformin. Angew. Chem., Int. Ed. 2014, 53, 5552-5555. (b) Blair, D. J.; Tanini, D.; Bateman, J. M.; Scott, H. K.; Myers, E. L.; Aggarwal, V. K. Selective Uni- and Bidirectional Homologation of Diborylmethane. Chem. Sci. 2017, 8, 2898-2903.

(22) Roesner, S.; Blair, D. J.; Aggarwal, V. K. Enantioselective Installation of Adjacent Tertiary Benzylic Stereocentres Using Lithiation-Borylation-Protodeboronation Methodology. Application to the Synthesis of Bifluranol and Fluorohexestrol. Chem. Sci. 2015, $6,3718-3723$.

(23) Clayden, J. In Organolithiums: Selectivity for Synthesis; Baldwin, J. E.; Williams, R. M., Eds.; Elsevier: Oxford, 2002; Vol. 23, pp 214-222.

(24) Odachowski, M.; Bonet, A.; Essafi, S.; Conti-Ramsden, P.; Harvey, J. N.; Leonori, D.; Aggarwal, V. K. Development of Enantiospecific Coupling of Secondary and Tertiary Boronic Esters with Aromatic Compounds. J. Am. Chem. Soc. 2016, 138, 95219532. 